ORIENTAL JOURNAL OF CHEMISTRY

An International Open Access, Peer Reviewed Research Journal

www.orientjchem.org
ISSN: 0970-020 X

CODEN: OJCHEG

2020, Vol. 36, No.(3):

Pg. 493-497

\title{
Development of Hydrogel from Rice Bran Starch
}

\section{LARCY MARIE O. PAGBILAO ${ }^{1,2}$, DANILET VI M. MENDOZA ${ }^{2 *}$, MARILENE C. HIPOLITO², JOCELYN P. GABRIEL ${ }^{2}$ and MARIO M. ABESAMIS, JR. ${ }^{2}$}

\author{
${ }^{1}$ Talavera National Highschool, Talavera, Nueva Ecija 3114, Philippines. \\ ${ }^{2}$ Chemistry and Environmental Science Department, College of Arts and Sciences, \\ Nueva Ecija University of Science and Technology, Cabanatuan City 3100, Philippines. \\ ${ }^{\star}$ Corresponding author E-mail: niletmendoza @ yahoo.com \\ http://dx.doi.org/10.13005/ojc/360319
}

(Received: May 10, 2020; Accepted: June 18, 2020)

\begin{abstract}
Wound dressing is a vital component of wound infection management specially by immunocompromised patients due to accidents that caused injuries. Biodegradable hydrogel-based wound dressings are being developed to acquire the advantage of high swelling capacity and being environment friendly at the same time. The study involves development of hydrogels using solution casting method with different ratio of rice bran starch. Mechanical properties such as tensile strength and swelling capacity were determined. The hydrogel developed with $7 \%$ starch emerged with comparable tensile strength and swelling capacity with the available wound dressing in the market. FT-IR spectra confirmed the crosslinking of PVA and starch while SEM images revealed that the hydrogel is not porous. Drug loading capacity of the hydrogel was determined to be $83.48 \%$ with PBS which is higher than with water. Results suggest that the developed hydrogel has great potential for wound dressing application with the advantage of being biodegradable.
\end{abstract}

Keywords: Hydrogel, Rice bran, Wound dressing, Biodegradable hydrogel, SW Fluid.

\section{INTRODUCTION}

Many people encountered accidents, burns, and other serious injuries that may lead to damage in skin and tissues which may lead to serious infections especially in the case of over aged people and other suffering from diseases that weakens their immune response to such. In these scenarios, they tend to have slow rate of skin degeneration and there is a need to have outside interventions for wound treatment and healing ${ }^{1}$. A wound can be described as a break in the skin resulting from thermal damage, physical damage or from physiological conditions ${ }^{2}$.

Wound dressing plays an important role in the entire management of wound infection ${ }^{3}$. Commonly used wound dressings are made up of hydrogels. Hydrogels are crosslinked polymeric materials with high water or liquid affinity ${ }^{4,5}$. Thus, these are commonly used as super-absorbent such as component of diapers ${ }^{6}$. Hydrogels that are available in the market are non-biodegradable that greatly contributes in increasing wastes in the

This is an Open Access article licensed under a Creative Commons license: Attribution 4.0 International (CC- BY). Published by Oriental Scientific Publishing Company @ 2018 
environment. Thus, developing natural polymer-based wound dressing is a topic of interest nowadays.

Starch is a biopolymer with high potential as material used in packaging technology and hydrogel preparation due to its low cost, good filmforming properties, biocompatibility and nontoxicity?. Even though starch show low mechanical strength and insufficient water resistance, it can be enhanced by mixing it with hydrophobic polymers like poly-vinyl alcohol (PVA). Addition of starch with PVA based gel greatly improves the quality of the hydrogel specially for wound dressing applications ${ }^{8,9}$. There are many factors in the development of hydrogels from starch raw materials that greatly affects its properties. Hydrogels as wound dressings should exhibit high tensile strength as well as swelling ability to be efficient. In the case of starch-based hydrogels, physicochemical properties are also influenced by amylose content while the swelling ability is due to the amylopectin ${ }^{10}$. Thus, biodegradation of this type of hydrogels are facilitated by alpha-amylase regulation ${ }^{11}$.

One source of relatively inexpensive rice starch which is a candidate given these properties, is rice bran. Rice bran is an industrial residue from milling process of rice grains. It contains vitamins, minerals, lipids, proteins and also high composition of starch in dry basis (defatted rice bran) ${ }^{12,13}$. Rice starch granules are small, ranging from 3 to $8 \mu \mathrm{m}$, and are polygonal in shape ${ }^{14}$.

The study is focused on the development and characterization of hydrogel from rice bran starch and assessment of its properties for wound dressing applications such as swelling ability and drug loading capacity.

\section{MATERIALS AND METHODS}

\section{Sample Collection and Preparation}

Rice bran was purchased from Philippine Rice Institute (PhilRice), Science City of Munoz, Nueva Ecija. The rice bran was defatted using chloroform as a solvent to reduce interference prior to the isolation of starch. Glycerol, polyvinylalcohol (PVA), glutaraldehyde, ethanol (95\%), sodium hydroxide and chloroform were purchased at Crismon Enterprises.

\section{Isolation of Starch from Defatted Rice bran}

Ten grams of defatted rice bran were soaked in $50 \mathrm{~mL}$ of distilled water at $30^{\circ} \mathrm{C}$ for 3 hours. The mixture was blended in a blender at $30^{\circ} \mathrm{C}$ for 5 minutes. A 60-mesh screen attached in the blender aided the separation of the extract from the rice bran when the extract was poured out of the blender. The rice bran was then blended once more with $70 \%$ ethanol and $0.1 \mathrm{M} \mathrm{NaOH}$ for $5 \mathrm{~min}$ for each solvent. The filtrate was centrifuged at $11,000 \times \mathrm{g}$ for 15 minutes. The residue was re-slurried, washed with deionized water and filtrated through a 200-mesh screen. The filtrate was filtered again using 2.5 $\mu \mathrm{m}$ filter paper and washed with $0.1 \mathrm{M} \mathrm{NaOH}$ and deionized water. The residue collected was dried at $60^{\circ} \mathrm{C}$ for 6 hours. The dried starch was ground with mortar and pestle and stored.

\section{Preparation of Starch-based hydrogel}

Hydrogel membranes were prepared using conventional solution casting method ${ }^{15}$. About 2.5 $\mathrm{g}$ of PVA was added to $50 \mathrm{~mL}$ of distilled water and the mixture was heated at $70^{\circ} \mathrm{C}$ for $2 \mathrm{~h}$ with constant stirring. Another $50 \mathrm{~mL}$ of distilled water was added to starch (5\%, $7 \%$ and $10 \% \mathrm{w} / \mathrm{v})$ and the resulting starch solutions were heated at $100^{\circ} \mathrm{C}$ for 15 min with constant stirring. Starch solutions were allowed to cool and were mixed with PVA solution with constant stirring to obtain homogeneous mixture. The chemical crosslinking agent solution was prepared by adding $0.5 \mathrm{~mL}$ of glutaraldehyde and $0.05 \mathrm{~mL}$ of $\mathrm{HCl}$ in $10 \mathrm{~mL}$ of ethanol. This mixture was added to the starch-PVA solution with constant stirring. About $2 \mathrm{~mL}$ of glycerol was then added with constant stirring and the solutions were sonicated for $2 \mathrm{~h}$ to obtain homogeneity. The resulting solutions were casted in molder and left overnight at room temperature to dry. The membranes were washed thoroughly with distilled water to wash off remaining $\mathrm{HCl}$ and glutaraldehyde. The membranes were then dried at room temperature.

\section{Determination of Mechanical Properties}

In order to determine the tensile strength of the membranes it was cut into pieces with $15 \times 200$ $\mathrm{mm}$ dimension. The thickness of the samples was measured before testing. Analysis was performed at a strain rate of $500 \mathrm{~mm} / \mathrm{min}$ at room temperature. The Instron Universal Testing Machine (ASTM D882) Model:5585H was used for mechanical testing. All mechanical tests are the mean of at least eight samples ${ }^{16}$. 
Swelling behavior of the hydrogels was measured against simulated wound fluid (SWF). Simulated wound fluid was prepared by taking $1.7 \mathrm{~g}$ of $\mathrm{NaCl}, 0.55 \mathrm{~g}$ of KCl, $7.5 \mathrm{~g}$ of $\mathrm{NaHCO}_{3}$ and $0.875 \mathrm{~g}$ of $\mathrm{NaH}_{2} \mathrm{PO}_{4}$ in $250 \mathrm{~mL}$ of distilled water. The $\mathrm{pH}$ of the prepared solution was found to be 8.0. To measure the swelling behaviour, the hydrogel membranes were cut into equal pieces of $2 \times 2 \mathrm{~cm}^{2}$ and weighed. Afterwards these pieces were immersed in SWF for 24 hours. After $24 \mathrm{~h}$, the pieces of hydrogel membranes were removed and their surface was cleaned with filter paper to remove any droplets and weighed again. The swelling ratio was then calculated based on Equation 1.

Swelling ratio $(\%)=\frac{\mathrm{Ws}-\mathrm{Wd}}{\mathrm{Wd}} \times 100$

Where Ws is the weight of the swelled membrane and $\mathrm{Wd}$ is the weight of the dried membrane.

The hydrogel sample with the highest tensile strength and swelling behavior was characterized using Fourier Transform Infrared Spectroscopy (FT-IR) and Scanning Electron Microscopy (SEM).

\section{Characterization of Hydrogel}

Rice bran starch, PVA and hydrogel membrane were subjected to FT-IR Spectroscopy. The spectra were recorded based on FT-IR spectra obtained using Shimadzu IR Prestige-21 spectrophotometer equipped with an Attenuated Total Reflection unit.

The surface morphology of the gel was investigated using Hitachi SEM Model TM300 at an acceleration voltage of $5 \mathrm{kV}$. The gel sample was mounted on aluminium stubs with double-sided adhesive tapes then the sample was coated with gold to avoid charging ${ }^{17}$.

\section{Drug Loading Capacity of Hydrogel}

The loading of drug was carried out by swelling equilibrium method and determined from the standard curves of the drug prepared at UV-Visible Spectrometer Lambda 365. The standard curves for Berberine hydrochloride was prepared at $\lambda_{\max }$ equal to $239 \mathrm{~nm}$ (in distilled water) and $\lambda_{\text {max }}$ of $348 \mathrm{~nm}$ (in Phosphate Buffer Saline (PBS)). The weighed hydrogel membranes were allowed to swell in the drug (Berberine hydrochloride) solution of known concentration $(1 \mathrm{mg} / \mathrm{mL})(\mathrm{Ci}) 24 \mathrm{~h}$ at $37^{\circ} \mathrm{C}$. The drug concentration (Cf) of the final solution was measured using UV-Vis Spectrometer ${ }^{18}$. Percentage drug loading capacity was calculated utilizing Equation 2.

$\%$ Drug Loading Capacity $=\frac{\mathrm{Ci}-\mathrm{Cf}}{\mathrm{Ci}} \times 100$

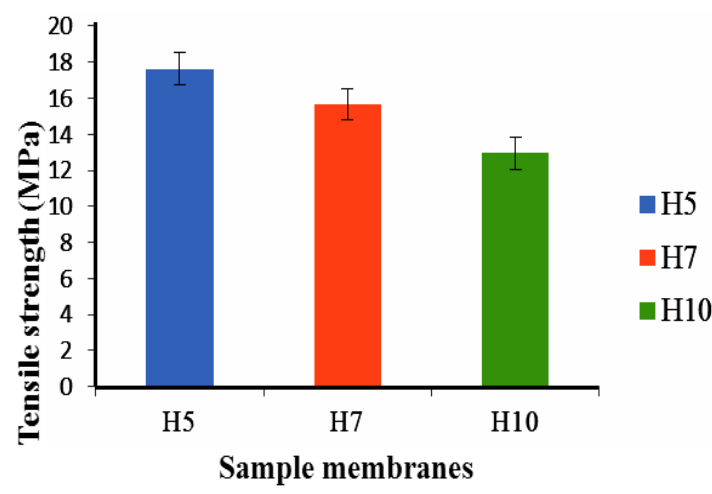

Fig. 1. Plot of tensile strength of the developed hydrogels. The error bars represent the standard deviation of measurements for 8 samples for each hydrogel

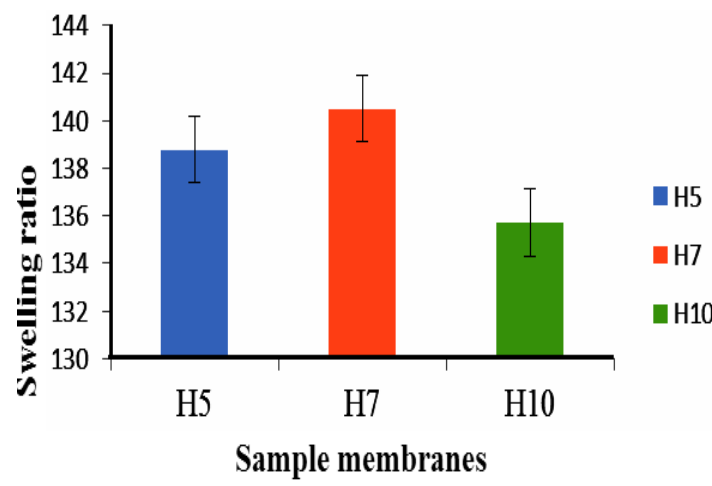

Fig. 2. Plot of swelling ratio of the developed hydrogels. The error bars represent the standard error of measurements for 8 samples for each hydrogel

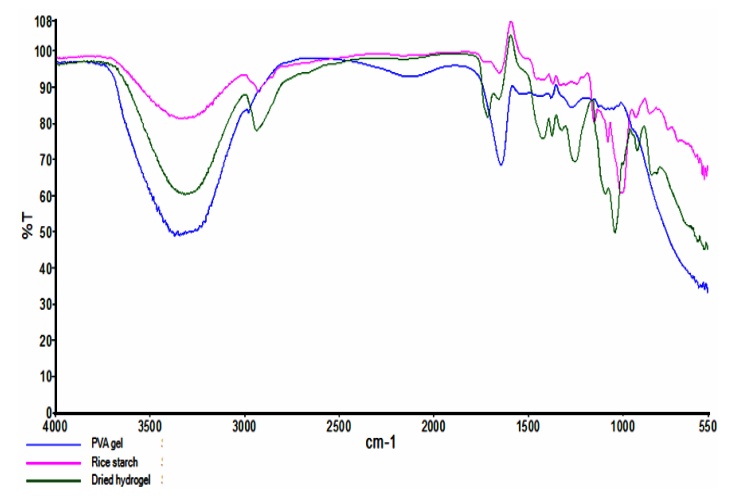

Fig. 3. FT-IR spectra of the developed hydrogel in comparison with pure PVA and rice starch 


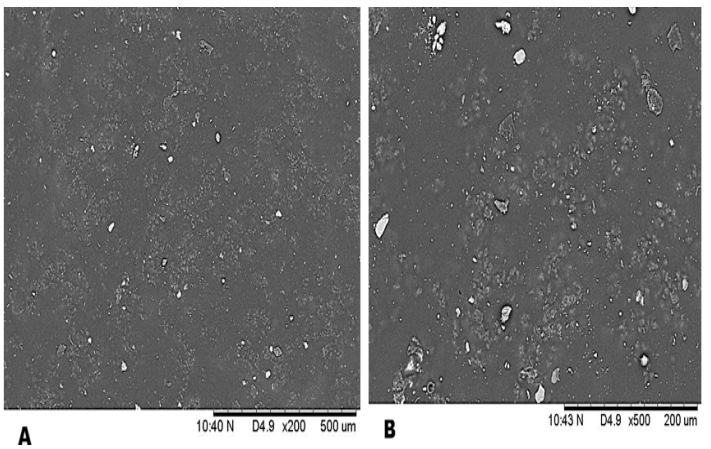

Fig. 4. SEM images of the developed hydrogel in 200x (A) and 500x (B) magnification

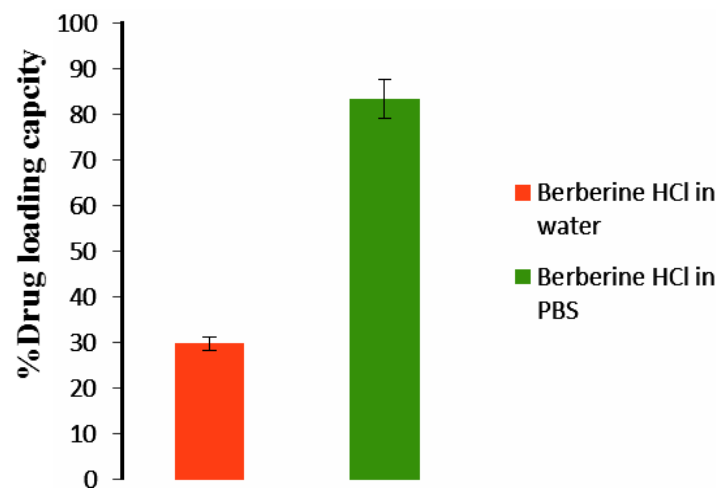

Fig. 5. Plot of \% Drug Loading Capacity of the developed hydrogels. The error bars represent the $5 \%$ errors of measurements for 8 samples for each hydrogel

\section{RESULTS DISCUSSION}

Rice bran was obtained from the variety of rice with high amylose content (NSIC Rc152). The isolated starch was $32 \%$ and used as a copolymer in preparation of hydrogel. Glutaraldehyde was used as the crosslinking agent in its preparation. The hydrogel membranes were prepared by solution casting method. The prepared hydrogels with different concentrations were designated as $\mathrm{H} 5$; for hydrogel with $5 \%$ starch solution, $\mathrm{H} 7$; for hydrogel with $7 \%$ starch solution, and $\mathrm{H} 10$; for the hydrogel with $10 \%$ starch solution. All samples for testing were dried in oven at $60^{\circ}$ for 6 hours.

\section{Mechanical Properties of the hydrogels}

Mechanical properties of the hydrogel are important factors to consider in its application as wound dressing. The tensile testing provides an indication of the strength of the hydrogel. The tensile strength of the hydrogel was compared to the tensile strength of the available hydrogel in the market as the positive control and measured using Universal Testing Machine (ASTM D882-09). $\mathrm{H} 5$ showed high tensile strength than hydrogel $\mathrm{H} 7$ and $\mathrm{H} 10$. The tensile strength of the hydrogels was determined to be $17.64 \pm 0.87 \mathrm{MPa}, 15.65 \pm 0.67$ $\mathrm{MPa}$ and $12.94 \pm 0.71 \mathrm{MPa}$ respectively (see Fig. 1), which are higher than the tensile strength of human skin, (11.5 MPa) based on the previous study ${ }^{19}$. While the tensile strength of the positive control was determined to be $19.64 \pm 0.75 \mathrm{MPa}$. The decreased in the tensile strength of the prepared hydrogels are due to the increasing amount of starch present in the membrane resulting in decreased in the chain length of the polyvinyl alcohol. Thus, the one with the least amount of starch emerged as having the highest tensile strength.

The use of hydrogels for the wound healing involves its direct contact with the body fluids. The characteristics of gels interaction with aqueous media were determined in simulated wound fluid (SWF). The swelling behavior of the hydrogels was measured by gravimetric method. It was observed that $\mathrm{H} 5$, $\mathrm{H} 7$ and $\mathrm{H} 10$ membranes have the high capacity in absorbing fluid which is more than $100 \% ; 138.78 \%$, $140.50 \%$ and $135.72 \%$ respectively (see Fig. 2). H7 showed comparable swelling capacity against the positive control with $140.53 \pm 0.26 \%$. This is due to the presence of hydroxyl groups in starch which helps in absorbing water and fluid. In the case of $\mathrm{H} 10$, the excess starch in the membrane was dissolved in the solution resulting in lower absorption of the membrane due to high solubility of starch with SWF.

\section{Characterization of hydrogels}

The hydrogel was characterized together with pure PVA and rice bran starch using FT-IR spectrum (see Fig. 3). It was observed that broad peaks at around $3300 \mathrm{~cm}^{-1}$ corresponding to $-\mathrm{OH}$ group appeared in all peaks. This hydroxyl group is responsible for the swelling capacity. Thus, this suggests that crosslinking of rice bran starch and PVA only slightly altered the hydroxyl groups still resulting in high swelling capacity. The peak at $2940 \mathrm{~cm}^{-1}$ indicates the presence of $\mathrm{sp}^{3}$ hybridized hydrocarbon chains. The peak that confirms the crosslinking of PVA and rice bran starch can be seen at $1714 \mathrm{~cm}^{-1}$.

Surface morphology of the hydrogel was investigated using SEM (see Fig. 4). It was observed that the hydrogel was not porous. Based 
on previous study, non-porous hydrogel can prevent the penetration of bacteria which can cause wound infection. Thus, the developed hydrogel can serve as barrier for microorganisms ${ }^{20}$.

\section{Drug Loading Capacity}

Berberine hydrochloride, a major alkaloid present in Ranunculaceae coptis, was an effective treatment for infections caused by Shigella and Escherichia $^{21}$. Berberine hydrochloride dissolved in water and PBS was loaded in the prepared hydrogel. The drug loading efficiency of the membrane was measured. The initial concentration of the drug loaded in the hydrogel membrane was $1 \mathrm{ppm}$. The final concentration of the drug solution was measured using the difference in absorbance reading upon subjecting it to UV-Vis spectrophotometer. The drug loading capacity of the hydrogel membrane in water and PBS was determined to be $29.90 \%$ and $83.48 \%$, respectively (see Figure 5).

\section{CONCLUSION}

Starch was isolated from rice bran using wet milling method. The isolated starch was $32 \%$ and used in preparation of hydrogel as copolymer with PVA. The developed hydrogel membranes were thin and flexible. The tensile strength of hydrogel membranes was measured and showed that all prepared membranes were higher than the reported tensile strength of human skin which is $11.5 \mathrm{MPa}$. Swelling capacity of the hydrogels showed that the membrane with $7 \%$ starch solution has a comparable swelling behavior with the positive control which is a commercially available wound dressing. FT-IR spectra of the hydrogel confirmed the crosslinking of starch and PVA while SEM images revealed that the hydrogel was not porous.

Drug loading capacity of the hydrogel membrane showed high absorption of Berberine hydrochloride observed in drug solution in PBS which is $83.48 \%$ than in drug dissolved in water which is $29.90 \%$. This suggests that $\mathrm{pH}$ greatly influenced the drug loading of the hydrogels. Thus, the developed hydrogel from rice bran starch has the properties suited for wound dressing applications.

\section{ACKNOWLEDGEMENT}

The authors wish to express their gratitude to Nueva Ecija University of Science and Technology administration, faculty and staff.

\section{Conflict of Interest}

The authors declare that there is no conflict of interest regarding the publication of this study.

\section{REFERENCES}

1. Hassan, A.; Naizi, M. B. K.; Hussain, A.; Farrukh, S.; Ahmad, T. JPE., 2017, 26, 235-243.

2. Onofrei, M. D.; Filimon, A. RNASR., 2016, 108-120.

3. Namazi, H.; Rakhshaei, R., Hamishehkar, H.; Kafil, H.S. IJBM., 2016, 85, 327-334.

4. Ahmed, E. M. JAR., 2013, 6(2), 105-121.

5. Fajardo, A. R.; Pereira, A. G. B.; Rubira, A.F.; Valente, A.J.M.; Muniz, E. C. Em., 2015, 1 , 319-360.

6. Peng, N.; Wang, Y.; Ye, Q.; Liang, L.; An, Y.; Li, Q.; Chang, C. CARBPOL., 2016, 137, 59-64.

7. Velasquez, D.; Pavon-Djavid, G.; Chaunier, L.; Meddahi-Pelle, A.; Lourdin, D. CARBPOL., 2015, 124, 180-187.

8. Kenawy, E.; Kamoun, E. A.; Eldin, M.S.M.; El-Meligy, M. A. Arab. J. Chem., 2014, 7(3), 372-380

9. Senna, M. M.; Mostafa, A. E. K. B.; Mahdy, S. R.; El-Naggar, A. W. M. Nucl Instrum Meth B., 2016, 386, 22-29.

10. Wani, A. A.; Singh, P.; Shah, M. A.; SchweiggertWeisz, U.; Gul, K.; Wani, I. A. Compr Rev Food Sci F., 2012, 11, 417-436.

11. Li, Juan; Mo, L.; Lu, C. H.; Fu, T.; Yang, H. H.;Tan,
W. Chem. Soc. Rev., 2016, 45(5), 1410-1431.

12. Capellini, M. C.; Giacomini, V.; Cuevas, M. S.; Rodrigues, C. E. Ind Crops Prod., 2017, 104, 133-143.

13. Sutanto, S.; Go, A. W.; Chen, K. H.; Ismadji, S.; Ju, Y. H. Waste Biomass Valori., 2017, 8(4), 1067-1080.

14. Fabian, C.; Ayucitra, A.; Ismadji, S.; Ju, Y.H. J Taiwan Inst Chem E., 2011, 42, 86-91.

15. Pal, K.; Banthia A. K.; Majumdar, D. K. Trends Biomater Artif Organs., 2006, 20, 59-67.

16. Tavakoli. J. J Mech Behav Biomed., 2017, 65 373-382.

17. Sanyang, M. L.; Sapuan, S. M.; Jawaid, M.; Ishak, M. R.; Sahari, J. JFST., 2015, 53(1), 326-336.

18. Singh, B.; Varshney, L.; Francis, S. Radiat Phys Chem., 2017, 135, 94-105.

19. Gallagher, A.J.; Ní Annaidh, A.; Bruyère, K. IRCOBI., 2012.

20. Yuk, H.; Zhang, T.; Lin, S.; Parada, G. A.; Zhao, X. Nat., 2016, 15(2), 190-196.

21. Zhao, L.; Li, Q.; Xu, X.; Kong, W.; Li, X.; Su, Y.; Gao, B. A. Chem. Eng. J., 2015, 287, 537-544. 